Алгебра и анализ

Toм 19 (2007), № 4
St. Petersburg Math. J.

Vol. 19 (2008), No. 4, Pages 561-575

S 1061-0022(08)01010-8

Article electronically published on May 9, 2008

\title{
MOTIVIC INTEGRALS AND FUNCTIONAL EQUATIONS
}

\author{
E. GORSKII
}

\begin{abstract}
A functional equation for the motivic integral corresponding to the Milnor number of an arc is derived by using the Denef-Loeser formula for the change of variables. Its solution is a function of five auxiliary parameters, it is unique up to multiplication by a constant, and there is a simple recursive algorithm to find its coefficients. The method is fairly universal and gives, for example, equations for the integral corresponding to the intersection number over the space of pairs of arcs and over the space of unordered collections of arcs.
\end{abstract}

\section{$\S 1$. INTRODUCTION}

Motivic integration, introduced by M. Kontsevich, is a powerful tool for exploring the space of formal arcs on a given variety. Motivic integrals provide the generating series for motivic measures of level sets of some arc invariants. There are examples of such integrals that can be calculated explicitly (see, e.g., [1]). In more general situations the values of such integrals are unknown; however, some of them satisfy functional equations if some auxiliary variables are introduced.

In this paper, with the help of the Denef-Loeser formula for the change of variables (see [1]), a functional equation is derived for the motivic integral that gives the generating series corresponding to the Milnor number of a plane curve. Its solution is unique up to multiplication by a constant, and there is a simple algorithm to express the solution's coefficients via the initial data. For example, this means that the solution satisfies some partial differential equations. The equation obtained gives a method for computing the motivic measure (and consequently, the Hodge-Deligne polynomial) of the stratum $\{\mu=$ const $\}$ in the space of plane curves. Some examples are considered in $\S 4$.

A similar idea gives some other equations, for example, an equation for the integral corresponding to the intersection number over the space of pairs of arcs. Moreover, using the notion of the power structure on the Grothendieck ring (see [2]), we introduce a motivic measure on the space of unordered collections of arcs. We also derive a curious equation for the integral corresponding to the intersection number in this case.

Some generating series with coefficients in the Grothendieck ring of varieties $K_{0}\left(\operatorname{Var}_{\mathbb{C}}\right)$ (or in the Grothendieck ring of Chow motives) satisfy functional equations similar to the functional equation for the Hasse-Weil zeta function. These equations, obtained by M. Kapranov [3] and F. Heinloth [4, follow from the duality theory on curves and Abelian varieties. It should be noted that they have an origin different from our approach.

\section{$\S 2$. Motivic Measure}

Let $\mathcal{L}=\mathcal{L}_{\mathbb{C}^{2}, 0}$ be the space of arcs at the origin on the plane. It is the set of pairs $(x(t), y(t))$ of formal power series (without degree 0 terms).

2000 Mathematics Subject Classification. Primary 32S45, $28 \mathrm{~B} 10$.

Key words and phrases. Motivic integration, Milnor number, motivic measure, Grothendieck ring.

Supported by the grant NSh-4719.2006.1. 
Let $K_{0}\left(\operatorname{Var}_{\mathbb{C}}\right)$ be the Grothendieck ring of quasiprojective complex algebraic varieties. It is generated by the isomorphism classes of complex quasiprojective algebraic varieties modulo the relations $[X]=[Y]+[X \backslash Y]$, where $Y$ is a Zariski closed subset of $X$. Multiplication is given by the formula $[X] \cdot[Y]=[X \times Y]$. Let $\mathbb{L} \in K_{0}\left(\operatorname{Var}_{\mathbb{C}}\right)$ be the class of the complex line.

Consider the ring $K_{0}\left(\operatorname{Var}_{\mathbb{C}}\right)\left[\mathbb{L}^{-1}\right]$ with the following filtration: $F_{k}$ is generated by the elements of the type $[X] \cdot\left[\mathbb{L}^{-n}\right]$ with $n-\operatorname{dim} X \geq k$. Let $\mathcal{M}$ be the completion of $K_{0}\left(\operatorname{Var}_{\mathbb{C}}\right)\left[\mathbb{L}^{-1}\right]$ corresponding to this filtration.

A set $A \subset \mathcal{L}$ is said to be cylindric if for some $N$ there exists a constructive subset $A_{N} \subset \mathcal{L}$ such that $A=\pi_{N}^{-1}\left(A_{N}\right)$. For cylindric sets, we introduce the motivic measure by the formula

$$
\chi_{g}(A)=\left[A_{N}\right] \mathbb{L}^{-2 N} \in K_{0}\left(\operatorname{Var}_{\mathbb{C}}\right)\left[\mathcal{L}^{-1}\right] .
$$

In [1], Denef and Loeser used some ideas of Kontsevich to construct a countably additive measure $\chi_{g}$ defined on a certain completion of the algebra of cylindric subsets of $\mathcal{L}$ and with values in the $\operatorname{ring} \mathcal{M}$. This measure allows one to naturally define the (motivic) integral for simple functions on $\mathcal{L}$ (see [1]).

In what follows, we shall often use the simple functions $v_{x}=\operatorname{Ord}_{0} x(t), v_{y}=\operatorname{Ord}_{0} y(t)$, and $v=\min \left\{v_{x}, v_{y}\right\}$, defined for an $\operatorname{arc} \gamma(t)=(x(t), y(t))$.

Let $h: Y \rightarrow X$ be a proper birational morphism of smooth manifolds of dimension $d$, and let $J=h^{*} K_{X}-K_{Y}$ be the relative canonical divisor on $Y$ (locally it is determined by the Jacobi determinant). It gives rise to a function ord ${ }_{J}$ on the space of $\operatorname{arcs}$ on $Y$; this function is the intersection number between the arc and the divisor. Then we have the following change of variables formula in the motivic integral (see [1]).

Theorem 1. Let $A$ be a measurable subset in the space of arcs on $X$, and let $\alpha$ be $a$ simple function. Then

$$
\int_{A} \alpha d \chi_{g}=\int_{h^{-1}(A)}\left(h^{*} \alpha\right) \mathbb{L}^{-\operatorname{ord}_{J}} d \chi_{g}
$$

If $h$ is a blow up of the origin in the plane, the relative canonical divisor coincides with the exceptional line, so that the function ord ${ }_{J}$ coincides with the intersection number with this line.

\section{§3. Functional Equation for the Milnor number}

The Milnor number of the plane curve given by the equation $\{f=0\}$ can be defined as the codimension of the ideal generated by the partial derivatives $\frac{\partial f}{\partial x}, \frac{\partial f}{\partial y}$.

We shall use the following statement.

Lemma 1. Suppose that, after blowing up the origin, the Milnor number of an irreducible curve is equal to $\mu$ and the intersection number with the exceptional divisor is equal to p. Then the Milnor number of the initial curve is equal to

$$
\mu+p(p-1)
$$

We put

$$
I(t, a, b, c, d, f)=\int_{\mathcal{L}} t^{\mu} a^{v_{x}} b^{v_{y}} c^{v_{x}^{2}} d^{v_{x} v_{y}} f^{v_{y}^{2}} d \chi_{g} .
$$

Theorem 2. This function satisfies the functional equation

$$
\begin{aligned}
I(t, a, b, c, d, f)= & I\left(t, t^{-1} a b \mathbb{L}^{-1}, b, t c d f, d f^{2}, f\right)+I\left(t, t^{-1} a b \mathbb{L}^{-1}, a, t c d f, d c^{2}, c\right) \\
& +I\left(t, t^{-1} a b \mathbb{L}^{-1}, 1, t c d f, 1,1\right) \cdot(\mathbb{L}-1) .
\end{aligned}
$$


Proof. Let

$$
A(t, a, b, c, d, f)=\int_{\left\{v_{y}>v_{x}\right\}} t^{\mu} a^{v_{x}} b^{v_{y}} c^{v_{x}^{2}} d^{v_{x} v_{y}} f^{v_{y}^{2}} d \chi_{g} .
$$

Observe that

$$
\int_{\left\{v_{x}>v_{y}\right\}} t^{\mu} a^{v_{x}} b^{v_{y}} c^{v_{x}^{2}} d^{v_{x} v_{y}} f^{v_{y}^{2}} d \chi_{g}=A(t, b, a, f, d, c) .
$$

We compute a similar integral over $\left\{v_{x}=v_{y}\right\}$. If $v_{x}=v_{y}$, then

$$
y=\lambda x+\widetilde{y}, \quad \lambda \neq 0, \quad v(\widetilde{y})>v(y) .
$$

For $\lambda$ fixed we have $\mu(x(t), y(t))=\mu(x(t), \widetilde{y}(t))$, whence

$$
\begin{aligned}
\int_{\left\{v_{x}=v_{y}\right\}} t^{\mu} a^{v_{x}} b^{v_{y}} c^{v_{x}^{2}} d^{v_{x} v_{y}} f^{v_{y}^{2}} d \chi_{g}=\int_{\left\{v_{x}=v_{y}\right\}} t^{\mu}(a b)^{v_{x}}(c d f)^{v_{x}^{2}} d \chi_{g} \\
=(\mathbb{L}-1) \int_{\left\{v_{x}<v_{\tilde{y}}\right\}} t^{\mu}(a b)^{v_{x}}(c d f)^{v_{x}^{2}} d \chi_{g}=A(t, a b, 1, c d f, 1,1) \cdot(\mathbb{L}-1) .
\end{aligned}
$$

Consequently,

$$
I(t, a, b, c, d, f)=A(t, a, b, c, d, f)+A(t, b, a, f, d, c)+A(t, a b, 1, c d f, 1,1) \cdot(\mathbb{L}-1) .
$$

Let us blow up the origin. If $v_{y}>v_{x}$, then $y(t)=x(t) \theta(t), \theta(0)=0$. Therefore, the corresponding modifications of the curves pass through the fixed point $p_{0}$ of the exceptional divisor related to the $x$-axis. Then

$$
\begin{gathered}
\sigma^{*} \mu=\mu+v_{x}\left(v_{x}-1\right), \quad \sigma^{*} v_{x}=v_{x}, \quad \sigma^{*} v_{x}^{2}=v_{x}^{2}, \\
\sigma^{*} v_{y}=v_{y}+v_{\theta}, \quad \sigma^{*} v_{x} v_{y}=v_{x}^{2}+v_{x} v_{\theta}, \quad \sigma^{*} v_{y}^{2}=v_{x}^{2}+2 v_{x} v_{\theta}+v_{\theta}^{2} .
\end{gathered}
$$

Using the Denef-Loeser change of variables formula, we obtain

$$
\begin{aligned}
A(t, a, b, c, d, f) & =\int_{\left\{v_{y}>v_{x}\right\}} t^{\mu} a^{v_{x}} b^{v_{y}} c^{v_{x}^{2}} d^{v_{x} v_{y}} f^{v_{y}^{2}} d \chi_{g} \\
& =\int_{\mathcal{L}} t^{\mu+v_{x}\left(v_{x}-1\right)} a^{v_{x}} b^{v_{x}+v_{\theta}} c^{v_{x}^{2}} d^{v_{x}^{2}+v_{x} v_{\theta}} f^{v_{x}^{2}+2 v_{x} v_{\theta}+v_{\theta}^{2}} \mathbb{L}^{-v_{x}} d \chi_{g} \\
& =\int_{\mathcal{L}} t^{\mu}\left(t^{-1} a b \mathbb{L}^{-1}\right)^{v_{x}} b^{v_{\theta}}(t c d f)^{v_{x}^{2}}\left(d f^{2}\right)^{v_{x} v_{\theta}} f^{v_{\theta}^{2}} d \chi_{g} \\
& =I\left(t, t^{-1} a b \mathbb{L}^{-1}, b, t c d f, d f^{2}, f\right) .
\end{aligned}
$$

Thus,

$$
\begin{aligned}
I(t, a, b, c, d, f)= & I\left(t, t^{-1} a b \mathbb{L}^{-1}, b, t c d f, d f^{2}, f\right)+I\left(t, t^{-1} a b \mathbb{L}^{-1}, a, t c d f, d c^{2}, c\right) \\
& +I\left(t, t^{-1} a b \mathbb{L}^{-1}, 1, t c d f, 1,1\right) \cdot(\mathbb{L}-1) .
\end{aligned}
$$

It is clear that

$$
I(t, a, b, c, d, f)=I(t, b, a, f, d, c),
$$

and differentiation under the integral yields

$$
-c \frac{\partial I}{\partial c}=\left[-a \frac{\partial}{\partial a}\right]^{2} I, \quad-d \frac{\partial I}{\partial d}=\left[a \frac{\partial}{\partial a}\right] \circ b \frac{\partial}{\partial b} I, \quad-f \frac{\partial I}{\partial f}=\left[-b \frac{\partial}{\partial b}\right]^{2} I .
$$

Theorem 3. A function $I(t, a, b, c, d, f)$ divisible by abcdf and satisfying the functional equation (11) and the symmetry condition (2) is unique up to multiplication by a constant. 
Before proving Theorem 3, we consider the following simpler example of a similar functional equation for the motivic integral. Let

$$
f(a, b)=\int_{\mathcal{L}} a^{v_{x}} b^{v_{y}} d \chi_{g}=\frac{a b \mathbb{L}^{-2}(\mathbb{L}-1)^{2}}{\left(1-a \mathbb{L}^{-1}\right)\left(1-b \mathbb{L}^{-1}\right)} .
$$

Much as above, we can use the change of variables formula to obtain the functional equation

$$
f(a, b)=f\left(a b \mathbb{L}^{-1}, a\right)+f\left(a b \mathbb{L}^{-1}, b\right)+f\left(a b \mathbb{L}^{-1}, 1\right) \cdot(\mathbb{L}-1) .
$$

We describe its solutions. Note that the definition of $f(a, b)$ implies that $f(a, b)=f(b, a)$ and $f(0, b)=0$. form

Let $f(a, b)=\sum_{i, j} f_{i j} a^{i} b^{j}$. Then the functional equation (4) can be rewritten in the

$$
\begin{aligned}
\sum_{i, j} f_{i j} a^{i} b^{j} & =\sum_{i, j} f_{i j} \mathbb{L}^{-i} a^{i+j} b^{i}+\sum_{i, j} f_{i j} \mathbb{L}^{-i} a^{i} b^{i+j}+(\mathbb{L}-1) \sum_{i, j} f_{i j} \mathbb{L}^{-i} a^{i} b^{i} \\
& =\sum_{i \geq j} f_{j, i-j} \mathbb{L}^{-j} a^{i} b^{j}+\sum_{i \leq j} f_{i, j-i} \mathbb{L}^{-i} a^{i} b^{j}+(\mathbb{L}-1) \sum_{i, j} f_{i j} \mathbb{L}^{-i} a^{i} b^{i} .
\end{aligned}
$$

Using the relations $f_{i j}=f_{j i}$ and $f_{i 0}=0$, we obtain a system of recurrence relations on the coefficients:

$$
\left\{\begin{array}{l}
f_{i j}=\mathbb{L}^{-j} f_{i-j, j} \\
f_{i i}=\mathbb{L}^{-i}(\mathbb{L}-1) \sum_{j=1}^{\infty} f_{i j} .
\end{array}\right.
$$

Below we shall use a more general system of equations:

$$
\left\{\begin{array}{l}
f_{i j}=\varepsilon_{j} f_{i-j, j} \\
f_{i i}=C \varepsilon_{i} \sum_{j=1}^{\infty} f_{i j} .
\end{array} \quad \text { if } \quad i>j,\right.
$$

Lemma 2. Suppose $1-\varepsilon_{i}-C \varepsilon_{i} \neq 0, C \neq 0, \varepsilon_{i} \neq 0$, and $\varepsilon_{i} \neq 1$ for all $i$. Then system (6) has a nonzero solution, which is determined uniquely up to multiplication by a constant and is such that $f_{i, j}=f_{j, i}$ and $f_{i, 0}=0$.

Proof. The first equation in (6) shows that

$$
\sum_{j>i} f_{i j}=\varepsilon_{i} \sum_{j>i} f_{i, j-i}=\varepsilon_{i} \sum_{j>0} f_{i, j} .
$$

Moreover, since $f_{i i}=C \varepsilon_{i} \sum_{j>0} f_{i j}$, we have $f_{i i}=C \sum_{j>i} f_{i j}$, whence

$$
\sum_{0<j<i} f_{i j}=\sum_{j>0} f_{i j}-f_{i i}-\sum_{j>i} f_{i j}=f_{i i}\left(\frac{1}{C \varepsilon_{i}}-1-\frac{1}{C}\right) .
$$

Thus,

$$
f_{i i}=\frac{C \varepsilon_{i}}{1-\varepsilon_{i}-C \varepsilon_{i}} \sum_{0<j<i} f_{i j} .
$$

Let $f_{11}$ be an arbitrary nonzero number. We compute $f_{i j}$. If $i \neq j$, we can use the first equation in (6), and for $i=j$ we can use the above identity. In any case, $f_{i j}$ will be expressed via $f_{k, l}$ with $k+l<i+j$, so that this process will stop and $f_{i j}$ will be expressed via $f_{11}$. Therefore, the solution is unique.

It is easy to prove that the algorithm described above gives indeed a solution of (흐). 
We prove Theorem 3. Consider equation (11). Let

$$
I(t, a, b, c, d, f)=\sum_{k_{1}, k_{2}, k_{3}, k_{4}, k_{5}} g_{k_{1}, k_{2}, k_{3}, k_{4}, k_{5}} a^{k_{1}} b^{k_{2}} c^{k_{3}} d^{k_{4}} f^{k_{5}} .
$$

Then

$$
\begin{aligned}
\sum_{\underline{k}} g_{\underline{k}} a^{k_{1}} b^{k_{2}} c^{k_{3}} d^{k_{4}} f^{k_{5}}= & \sum_{k_{1}, k_{2}, k_{3}, k_{4}, k_{5}} g_{k_{1}, k_{2}, k_{3}, k_{4}, k_{5}}\left(t^{-1} \mathbb{L}^{-1} a b\right)^{k_{1}} b^{k_{2}}(t c d f)^{k_{3}}\left(d f^{2}\right)^{k_{4}} f^{k_{5}} \\
& +\sum_{k_{1}, k_{2}, k_{3}, k_{4}, k_{5}} g_{k_{1}, k_{2}, k_{3}, k_{4}, k_{5}}\left(t^{-1} \mathbb{L}^{-1} a b\right)^{k_{1}} a^{k_{2}}(t c d f)^{k_{3}}\left(d c^{2}\right)^{k_{4}} c^{k_{5}} \\
& +(\mathbb{L}-1) \cdot \sum_{k_{1}, k_{2}, k_{3}, k_{4}, k_{5}} g_{k_{1}, k_{2}, k_{3}, k_{4}, k_{5}}\left(t^{-1} \mathbb{L}^{-1} a b\right)^{k_{1}}(t c d f)^{k_{3}} .
\end{aligned}
$$

We obtain the following system of equations:

$$
\left\{\begin{aligned}
& g_{k_{1}, k_{2}, k_{3}, k_{4}, k_{5}}=t^{k_{3}-k_{1}} \mathbb{L}^{-k_{1}} g_{k_{1}, k_{2}-k_{1}, k_{3}, k_{4}-k_{3}, k_{5}-2 k_{4}+k_{3}} \quad \text { if } k_{2}>k_{1}, k_{4}>k_{3}, k_{5}>2 k_{4}-k_{3}, \\
& g_{k_{1}, k_{1}, k_{3}, k_{3}, k_{3}}=t^{k_{3}-k_{1}} \mathbb{L}^{-k_{1}}(\mathbb{L}-1) \sum_{k_{2}, k_{4}, k_{5}} g_{k_{1}, k_{2}, k_{3}, k_{4}, k_{5}} \\
& g_{k_{1}, k_{2}, k_{3}, k_{4}, k_{5}}=g_{k_{2}, k_{1}, k_{5}, k_{4}, k_{3} .}
\end{aligned}\right.
$$

Moreover, $g_{k_{1}, k_{2}, k_{3}, k_{4}, k_{5}}=0$ if the collection $\left(k_{1}, k_{2}, k_{3}, k_{4}, k_{5}\right)$ does not satisfy the inequalities

Or

$$
k_{2} \geq k_{1}, \quad k_{4} \geq k_{3}, \quad k_{5} \geq 2 k_{4}-k_{3}
$$

$$
k_{1} \geq k_{2}, \quad k_{4} \geq k_{5}, \quad k_{3} \geq 2 k_{4}-k_{5}
$$

As in the proof of Lemma 2, we can check that any coefficient can be expressed in terms of $g_{1,1,1,1,1}$ and the solution is unique.

Since, by Theorem $2, \int_{\mathcal{L}} t^{\mu} a^{v_{x}} b^{v_{y}} c^{v_{x}^{2}} d^{v_{x} v_{y}} f^{v_{y}^{2}} d \chi_{g}$ satisfies equation (11), every solution of (11) is proportional to this solution.

Therefore,

$$
I(t, a, b, c, d, f)=\sum G_{i, j}(t) a^{i} b^{j} c^{i^{2}} d^{i j} e^{j^{2}} .
$$

Thus, (77) implies the partial differential equations (3).

We compute $g_{1,1,1,1,1}$. If $v_{x}=v_{y}=1$, then $\mu=0$, whence

$$
g_{1,1,1,1,1}=\chi_{g}\left\{v_{x}=v_{y}=1\right\}=(\mathbb{L}-1)^{2} \mathbb{L}^{-2} .
$$

\section{$\S 4$. EXAMPLES}

Recall that

$$
G_{i, j}(t)=\int_{v_{x}=i, v_{y}=j} t^{\mu} d \chi_{g}
$$

It can be checked that system (77) gives a system of equations for $G_{i, j}(t)$ of the form (6) with

$$
\varepsilon_{k}=t^{k^{2}-k} \mathbb{L}^{-k}, \quad C=(\mathbb{L}-1) .
$$

Therefore, by the proof of Lemma 2, we have

$$
\begin{cases}G_{i, j}(t)=t^{j^{2}-j} \mathbb{L}^{-j} G_{i-j, j} & \text { if } j<i \\ G_{i, i}(t)=\frac{(\mathbb{L}-1) t^{i^{2}-i}}{1-t^{2}-i} \sum_{j<i} G_{i, j}(t) & \\ G_{1,1}(t)=(\mathbb{L}-1)^{2} \mathbb{L}^{-2} & \end{cases}
$$

Then $G_{1, n}(t)=\mathbb{L}^{-1} G_{1, n-1}$, whence

$$
G_{1, n}(t)=(\mathbb{L}-1)^{2} \mathbb{L}^{-1-n} .
$$


This corresponds to the fact that every arc with $v_{x}=1$ is smooth; thus, $\mu=0$.

Moreover, since $G_{2, n}(t)=t^{2} \mathbb{L}^{-2} G_{2, n-2}(t)$ for $n>2$, we have

$$
G_{2,2 n-1}=t^{2 n-2} \mathbb{L}^{2-2 n} G_{2,1}=(\mathbb{L}-1)^{2} t^{2 n-2} \mathbb{L}^{-1-2 n} .
$$

The Milnor number of every arc with $v_{x}=2$ and $v_{y}$ odd is equal to $v_{y}-1$, because the singularity is of $A_{v_{y}-1}$ type.

In the case where $v_{y}$ is even, the result is more interesting:

$$
\begin{aligned}
G_{2,2 n}(t) & =t^{2 n-2} \mathbb{L}^{2-2 n} G_{2,2}=t^{2 n} \mathbb{L}^{-2 n} \frac{(\mathbb{L}-1)}{1-t^{2} \mathbb{L}^{-1}} G_{1,2}(t) \\
& =\frac{t^{2 n} \mathbb{L}^{-2 n-3}(\mathbb{L}-1)^{3}}{1-t^{2} \mathbb{L}-1}=\sum_{k=0}^{\infty} t^{2(n+k)} \mathbb{L}^{-2 n-3-k}(\mathbb{L}-1)^{3} .
\end{aligned}
$$

We explain this answer. Making a change of variables, we may assume that $x(t)=t^{2}$. Let

$$
y(t)=a_{2 n} t^{2 n}+\cdots+a_{2 m+1} t^{2 m+1}+\cdots,
$$

where $a_{2 m+1}$ is the first nonzero coefficient with odd index. Then the equation of this curve is

$$
F(x, y)=x^{2 m+1}+\left(y-a_{2 n} x^{n}-\cdots-a_{2 m} x^{m}\right)^{2}+\cdots=0,
$$

so that the Milnor number is equal to $2 m$. Put $k=m-n$. For fixed $x(t)$, the measure of the set of series $y(t)$ with given $m$ is equal to

$$
(\mathbb{L}-1)^{2} \mathbb{L}^{-2 n-(m-n+1}=(\mathbb{L}-1)^{2} \mathbb{L}^{-2 n-k-1},
$$

and the measure of the set of series $x(t)$ with order 2 is equal to $(\mathbb{L}-1) \mathbb{L}^{-2}$. Multiplying these expressions, we obtain the above formula.

Lemma 3. Let a be the greatest common divisor of $i$ and $j$. Then

$$
G_{i, j}(t)=t^{(i-1)(j-1)-(a-1)^{2}} \mathbb{L}^{2 a-i-j} G_{a, a}(t) .
$$

Proof. For $i=j$ the formula is tautological. Suppose it is true for $i-j$ and $j(i>j)$. Then

$$
G_{i, j}(t)=t^{j^{2}-j} \mathbb{L}^{-j} G_{i-j, j}=t^{(i-j-1)(j-1)-(a-1)^{2}+j(j-1)} \mathbb{L}^{2 a-(i-j)-j-j} G_{a, a}(t) .
$$

Now the statement follows from the Euclid algorithm.

The following answers can be obtained in a similar way:

\begin{tabular}{|c|c|}
\hline$a$ & $G_{a, a}(t)$ \\
\hline 1 & $(\mathbb{L}-1) \mathbb{L}^{2}$ \\
\hline 2 & $\frac{(\mathbb{L}-1)^{3} t^{2} \mathbb{L}^{-5}}{1-t^{2} \mathbb{L}^{-1}}$ \\
\hline 3 & $\frac{(\mathbb{L}-1)^{3} t^{6} \mathbb{L}^{-7}\left(1+t^{2} \mathbb{L}^{-1}\right)}{1-t^{6} \mathbb{L}^{-2}}$ \\
\hline 4 & $\frac{(\mathbb{L}-1)^{3} t^{12} \mathbb{L}^{-9}\left(1-t^{2} \mathbb{L}^{-1}+t^{6} \mathbb{L}^{-1}-t^{8} \mathbb{L}^{-3}\right)}{\left(1-t^{12} \mathbb{L}^{-3}\right)\left(1-t^{2} \mathbb{L}^{-1}\right)}$ \\
\hline
\end{tabular}

Together with Lemma 3 , this table provides $G_{i, j}(t)$ with $\operatorname{gcd}(i, j) \leq 4$. 
Proposition. Let $a=\operatorname{gcd}(i, j) . G_{i j}(t)$ is a power series in $t$ whose coefficients are Laurent polynomials in $\mathbb{L}$. If $a=1$, then

$$
G_{i, j}(t)=(\mathbb{L}-1)^{2} t^{(i-1)(j-1)} \mathbb{L}^{-i-j}
$$

and

$$
G_{i, j}(t)=(\mathbb{L}-1)^{3} t^{(i-1)(j-1)+a-1} \mathbb{L}^{-i-j-1}+\text { terms of higher degree in } t .
$$

Proof. The first statement can easily be checked by induction. The case where $a=1$ follows from Lemma 3. We prove the formula for $a>1$. We have

$$
\begin{aligned}
G_{a, a}(t) & =\frac{(\mathbb{L}-1) t^{a^{2}-a} \mathbb{L}^{-a}}{1-t^{a^{2}-a} \mathbb{L}^{1-a}}\left(G_{a, 1}(t)+\mathrm{O}(t)\right) \\
& =(\mathbb{L}-1) t^{a^{2}-a} \mathbb{L}^{-a}(1+\mathrm{O}(t))\left((\mathbb{L}-1)^{2} \mathbb{L}^{-a-1}+\mathrm{O}(t)\right) \\
& =(\mathbb{L}-1)^{3} t^{a^{2}-a} \mathbb{L}^{-2 a-1}+\mathrm{O}\left(t^{a^{2}-a+1}\right) .
\end{aligned}
$$

Now the statement follows from Lemma 3.

\section{§5. Functional EQuation for the intersection Number}

Let

$$
\begin{aligned}
& J(t, a, b, c, d, p, q, r, s) \\
& \quad=\int_{\mathcal{L}^{(1)} \times \mathcal{L}^{(2)}} t^{\gamma_{1} \circ \gamma_{2}} a^{v_{x}^{(1)} v_{x}^{(2)}} b^{v_{x}^{(1)}} v_{y}^{(2)} c^{v_{y}^{(1)}} v_{x}^{(2)} d^{v_{y}^{(1)}} v_{y}^{(2)} p^{v_{x}^{(1)}} q_{y}^{v_{y}^{(1)}} r^{v_{x}^{(2)}} s^{v_{y}^{(2)}} d \chi_{g} \\
& \quad B(t, a, b, c, d, p, q, r, s) \\
& \quad=\int_{\left\{v_{y}^{(1)}>v_{x}^{(1)}, v_{y}^{(2)}>v_{x}^{(2)}\right\}} t^{\gamma_{1} \circ \gamma_{2}} a^{v_{x}^{(1)} v_{x}^{(2)}} b^{v_{x}^{(1)} v_{y}^{(2)}} c^{v_{y}^{(1)}} v_{x}^{(2)} d_{y}^{v_{y}^{(1)} v_{y}^{(2)}} p^{v_{x}^{(1)}} q_{y}^{v_{y}^{(1)}} r^{v_{x}^{(2)}} s^{v_{y}^{(2)}} d \chi_{g}
\end{aligned}
$$

Note that

$$
\sigma\left(\gamma_{1}\right) \circ \sigma\left(\gamma_{2}\right)=\gamma_{1} \circ \gamma_{2}+v_{1} v_{2}
$$

Therefore, if after blowing up the origin two arcs intersect the exceptional divisor at different points, their intersection number is equal to the product of multiplicities.

We split $\mathcal{L}^{(1)} \times \mathcal{L}^{(2)}$ into components corresponding to inequalities between $v_{y}^{(1)}$ and $v_{x}^{(1)}$, and also $v_{y}^{(2)}$ and $v_{x}^{(2)}$.

1) $v_{y}^{(1)}>v_{x}^{(1)}, v_{y}^{(2)}>v_{x}^{(2)}$. By definition, the integral is equal to

$$
B(t, a, b, c, d, p, q, r, s) \text {. }
$$

2) $v_{y}^{(1)}>v_{x}^{(1)}, v_{y}^{(2)}<v_{x}^{(2)}$. Since after blow up the arcs intersect the exceptional divisor at different points, the integral is equal to

$$
\begin{aligned}
\left.\int_{\left\{v_{y}^{(1)}\right.}>v_{x}^{(1)}, v_{y}^{(2)}<v_{x}^{(2)}\right\} & t^{v_{x}^{(1)} v_{y}^{(2)}} a^{v_{x}^{(1)} v_{x}^{(2)}} b_{x}^{v_{x}^{(1)} v_{y}^{(2)}} c^{v_{y}^{(1)} v_{x}^{(2)}} d^{v_{y}^{(1)} v_{y}^{(2)}} p_{x}^{v_{x}^{(1)}} q^{v_{y}^{(1)}} r_{x}^{v_{x}^{(2)}} s^{v_{y}^{(2)}} d \chi_{g} \\
= & \left(v_{x}^{(1)}=i, v_{y}^{(1)}=j, v_{y}^{(2)}=k, v_{x}^{(2)}=l\right) \\
& =\sum_{i<j, k<l} a^{i l}(b t)^{i k} c^{j l} d^{j k} p^{i} q^{j} r^{l} s^{k}(\mathbb{L}-1)^{4} \mathbb{L}^{-i-j-k-l} \\
& =(\mathbb{L}-1)^{4} \Phi\left(b t, a, d, c ; \mathbb{L}^{-1} p, \mathbb{L}^{-1} q, \mathbb{L}^{-1} s, \mathbb{L}^{-1} r\right),
\end{aligned}
$$

where $\Phi$ is defined by the equation

$$
\Phi(\alpha, \beta, \gamma, \delta, \pi, \kappa, \rho, \sigma)=\sum_{i<j, k<l} \alpha^{i k} \beta^{i l} \gamma^{j k} \delta^{j l} \pi^{i} \kappa^{j} \rho^{k} \sigma^{l} .
$$


3) $v_{y}^{(1)}>v_{x}^{(1)}, v_{y}^{(2)}=v_{x}^{(2)}$. After blowing up, the arcs intersect the exceptional divisor at different points. Therefore, the integral is equal to

$$
\begin{aligned}
\int_{\left\{v_{y}^{(1)}>v_{x}^{(1)}, v_{y}^{(2)}=v_{x}^{(2)}\right\}} t_{x}^{v_{x}^{(1)} v_{x}^{(2)}} a^{v_{x}^{(1)} v_{x}^{(2)}} b_{x}^{v_{x}^{(1)} v_{x}^{(2)}} c^{v_{y}^{(1)} v_{x}^{(2)}} d_{y}^{v_{y}^{(1)} v_{x}^{(2)}} p^{v_{x}^{(1)}} q^{v_{y}^{(1)}} r_{x}^{v_{x}^{(2)}} s^{v_{x}^{(2)}} d \chi_{g} \\
=\sum_{i<j, k}(t a b)^{i k}(c d)^{j k} p^{i} q^{j}(r s)^{k}(\mathbb{L}-1)^{4} \mathbb{L}^{-i-j-2 k} \\
=(\mathbb{L}-1)^{4} \Psi\left(t a b, c d, p \mathbb{L}^{-1}, q \mathbb{L}^{-1}, r s \mathbb{L}^{-2}\right),
\end{aligned}
$$

where $\Psi$ is defined by the equation

$$
\Psi(\alpha, \beta, \pi, \kappa, \rho)=\sum_{i<j, k} \alpha^{i k} \beta^{j k} \pi^{i} \kappa^{j} \rho^{k} .
$$

4) $v_{y}^{(1)}<v_{x}^{(1)}, v_{y}^{(2)}>v_{x}^{(2)}$. Then

$$
\begin{aligned}
& \int_{\left\{v_{y}^{(1)}<v_{x}^{(1)}, v_{y}^{(2)}>v_{x}^{(2)}\right\}} t^{v_{y}^{(1)} v_{x}^{(2)}} a^{v_{x}^{(1)} v_{x}^{(2)}} b^{v_{x}^{(1)} v_{y}^{(2)}} c^{v_{y}^{(1)}} v_{x}^{(2)} d^{v_{y}^{(1)}} v_{y}^{(2)} p^{v_{x}^{(1)}} q_{y}^{v_{y}^{(1)}} r^{v_{x}^{(2)}} s^{v_{y}^{(2)}} d \chi_{g} \\
& =\sum_{i<j, k<l} a^{j k} b^{j l}(c t)^{i k} d^{i l} p^{j} q^{i} r^{k} s^{l}(\mathbb{L}-1)^{4} \mathbb{L}^{-i-j-k-l} \\
& =(\mathbb{L}-1)^{4} \Phi\left(c t, d, a, b, q \mathbb{L}^{-1}, p \mathbb{L}^{-1}, r \mathbb{L}^{-1}, s \mathbb{L}^{-1}\right) .
\end{aligned}
$$

5) $v_{y}^{(1)}<v_{x}^{(1)}, v_{y}^{(2)}<v_{x}^{(2)}$. By symmetry, it is clear that the integral is equal to

$$
\begin{aligned}
& \int_{\left\{v_{y}^{(1)}>v_{x}^{(1)}, v_{y}^{(2)}>v_{x}^{(2)}\right\}} t^{\gamma_{1} \circ \gamma_{2}} a_{y}^{v_{y}^{(1)} v_{y}^{(2)}} b_{y}^{v_{y}^{(1)} v_{x}^{(2)}} c^{v_{x}^{(1)} v_{y}^{(2)}} d^{v_{x}^{(1)} v_{x}^{(2)}} p^{v_{y}^{(1)}} q^{v_{x}^{(1)}} r_{y}^{v_{y}^{(2)}} s^{v_{x}^{(2)}} d \chi_{g} \\
& =B(t, d, b, c, a, q, p, s, r) \text {. }
\end{aligned}
$$

6) $v_{y}^{(1)}<v_{x}^{(1)}, v_{y}^{(2)}=v_{x}^{(2)}$. Then

$$
\begin{aligned}
\left.\int_{\left\{v_{y}^{(1)}\right.}<v_{x}^{(1)}, v_{y}^{(2)}=v_{x}^{(2)}\right\} & t^{v_{y}^{(1)} v_{x}^{(2)}} a^{v_{x}^{(1)} v_{x}^{(2)}} b_{x}^{v_{x}^{(1)} v_{x}^{(2)}} c^{v_{y}^{(1)} v_{x}^{(2)}} d^{v_{y}^{(1)} v_{x}^{(2)}} p^{v_{x}^{(1)}} q^{v_{y}^{(1)}} r_{x}^{v_{x}^{(2)}} s^{v_{x}^{(2)}} d \chi_{g} \\
& =\sum_{i<j, k}(t c d)^{i k}(a b)^{j k} p^{j} q^{i}(r s)^{k}(\mathbb{L}-1)^{4} \mathbb{L}^{-i-j-2 k} \\
& =(\mathbb{L}-1)^{4} \Psi\left(t c d, a b, q \mathbb{L}^{-1}, p \mathbb{L}^{-1}, r s \mathbb{L}^{-2}\right) .
\end{aligned}
$$

7) $v_{y}^{(1)}=v_{x}^{(1)}, v_{y}^{(2)}>v_{x}^{(2)}$. Then

$$
\begin{aligned}
\int_{\left\{v_{y}^{(1)}\right.} & \left.=v_{x}^{(1)}, v_{y}^{(2)}>v_{x}^{(2)}\right\} \\
& =\sum_{i<j, k}(t b d)^{i k}(a c)^{j k}(p q)^{k} r^{i} s^{j}(\mathbb{L}-1)^{4} \mathbb{L}^{-i-j-2 k} a_{x}^{v^{(1)}} v_{x}^{(2)} b_{x}^{v_{x}^{(1)}} v_{y}^{(2)} c^{v_{x}^{(1)} v_{x}^{(2)}} d^{v_{x}^{(1)} v_{y}^{(2)}} p_{x}^{v_{x}^{(1)}} q^{v_{x}^{(1)}} r_{x}^{v_{x}^{(2)}} s^{v_{y}^{(2)}} d \chi_{g} \\
& =(\mathbb{L}-1)^{4} \Psi\left(t a c, b d, r \mathbb{L}^{-1}, s \mathbb{L}^{-1}, p q \mathbb{L}^{-2}\right) .
\end{aligned}
$$

8) $v_{y}^{(1)}=v_{x}^{(1)}, v_{y}^{(2)}<v_{x}^{(2)}$. Then

$$
\begin{aligned}
\int_{\left\{v_{y}^{(1)}\right.} & \left.=v_{x}^{(1)}, v_{y}^{(2)}<v_{x}^{(2)}\right\} \\
& =\sum_{i<j, k}(t b d)^{v_{x}^{(1)}}(a c)^{j k} r^{j} s^{i}(p q)^{k}(\mathbb{L}-1)^{4} \mathbb{L}^{-i-j-2 k} v_{x}^{(2)} b^{v_{x}^{(1)}} v_{y}^{(2)} c^{v_{x}^{(1)} v_{x}^{(2)}} d^{v_{x}^{(1)} v_{y}(2)} p^{v_{x}^{(1)}} q^{v_{x}^{(1)}} r_{x}^{v_{x}^{(2)}} s^{v_{y}^{(2)}} d \chi_{g} \\
& =(\mathbb{L}-1)^{4} \Psi\left(t b d, a c, s \mathbb{L}^{-1}, r \mathbb{L}^{-1}, p q \mathbb{L}^{-2}\right) .
\end{aligned}
$$


9) $v_{y}^{(1)}=v_{x}^{(1)}, v_{y}^{(2)}=v_{x}^{(2)}$. In this case we have

$$
\begin{cases}y^{(1)}=\lambda_{1} x^{(1)}+\widetilde{y}^{(1)}, & v_{\widetilde{y}}^{(1)}>v_{x}^{(1)}, \\ y^{(2)}=\lambda_{2} x^{(2)}+\widetilde{y}^{(2)}, & v_{\widetilde{y}}^{(2)}>v_{x}^{(2)},\end{cases}
$$

$\lambda_{1} \neq 0, \lambda_{2} \neq 0$.

a) $\lambda_{1} \neq \lambda_{2}$. Then

$$
\begin{aligned}
{\left[\left\{\lambda_{1}, \lambda_{2} \in \mathbb{C}^{*} \mid \lambda_{1} \neq \lambda_{2}\right\}\right] } & =\left[\mathbb{C}^{*}\right]^{2}-\left[\left\{\lambda_{1}, \lambda_{2} \in \mathbb{C}^{*} \mid \lambda_{1}=\lambda_{2}\right\}\right] \\
& =(\mathbb{L}-1)^{2}-(\mathbb{L}-1)=(\mathbb{L}-1)(\mathbb{L}-2),
\end{aligned}
$$

and for fixed $\left(\lambda_{1}, \lambda_{2}\right)$ the integral is equal to

$$
\begin{aligned}
\int_{\left\{v_{\tilde{y}}^{(1)}>v_{x}^{(1)}, v_{\tilde{y}}^{(2)}>v_{x}^{(2)}\right\}} t^{v_{x}^{(1)} v_{x}^{(2)}} a^{v_{x}^{(1)} v_{x}^{(2)}} b^{v_{x}^{(1)} v_{x}^{(2)}} c^{v_{x}^{(1)} v_{x}^{(2)}} d^{v_{x}^{(1)} v_{x}^{(2)}} p^{v_{x}^{(1)}} q^{v_{x}^{(1)}} r_{x}^{v_{x}^{(2)}} s^{v_{x}^{(2)}} d \chi_{g} \\
\quad=\sum_{i<j, k<l}(t a b c d)^{i k}(p q)^{i}(r s)^{k}(\mathbb{L}-1)^{4} \mathbb{L}^{-i-j-k-l} \\
=(\mathbb{L}-1)^{4} \Phi\left(t a b c d, 1,1,1 ; \mathbb{L}^{-1} p q, \mathbb{L}^{-1}, \mathbb{L}^{-1} r s, \mathbb{L}^{-1}\right) .
\end{aligned}
$$

Therefore, the contribution of the stratum $\left\{v_{x}^{(1)}=v_{y}^{(1)}, v_{x}^{(2)}=v_{y}^{(2)}, \lambda_{1} \neq \lambda_{2}\right\}$ is equal to

$$
(\mathbb{L}-1)^{5}(\mathbb{L}-2) \Phi\left(\text { tabcd }, 1,1,1 ; \mathbb{L}^{-1} p q, \mathbb{L}^{-1}, \mathbb{L}^{-1} r s, \mathbb{L}^{-1}\right) .
$$

b) $\lambda_{1}=\lambda_{2}=\lambda$. Since the intersection number does not change under the affine change of variables $(x, y) \mapsto(x, y-\lambda x)$, for fixed $\lambda$ the integral is equal to

$$
\begin{aligned}
& \int_{\left\{v_{\tilde{y}}^{(1)}>v_{x}^{(1)}, v_{\tilde{y}}^{(2)}>v_{x}^{(2)}\right\}} t^{\gamma_{1} \circ \gamma_{2}} a^{v_{x}^{(1)} v_{x}^{(2)}} b^{v_{x}^{(1)} v_{x}^{(2)}} c^{v_{x}^{(1)} v_{x}^{(2)}} d^{v_{x}^{(1)} v_{x}^{(2)}} p^{v_{x}^{(1)}} q^{v_{x}^{(1)}} r_{x}^{v_{x}^{(2)}} s^{v_{x}^{(2)}} d \chi_{g} \\
& \quad=B(t, a b c d, 1,1,1, p q, 1, r s, 1) .
\end{aligned}
$$

Consequently, the total contribution of this stratum is equal to

$$
(\mathbb{L}-1) B(t, a b c d, 1,1,1, p q, 1, r s, 1) .
$$

So, we have

$$
\begin{aligned}
J(t, a, b, c, d, & p, q, r, s) \\
= & B(t, a, b, c, d, p, q, r, s)+(\mathbb{L}-1)^{4} \Phi\left(b t, a, d, c ; \mathbb{L}^{-1} p, \mathbb{L}^{-1} q, \mathbb{L}^{-1} s, \mathbb{L}^{-1} r\right) \\
& +(\mathbb{L}-1)^{4} \Psi\left(t a b, c d, p \mathbb{L}^{-1}, q \mathbb{L}^{-1}, r s \mathbb{L}^{-2}\right) \\
& +(\mathbb{L}-1)^{4} \Phi\left(c t, d, a, b, q \mathbb{L}^{-1}, p \mathbb{L}^{-1}, r \mathbb{L}^{-1}, s \mathbb{L}^{-1}\right) \\
& +B(t, d, c, b, a, q, p, s, r)+(\mathbb{L}-1)^{4} \Psi\left(t c d, a b, q \mathbb{L}^{-1}, p \mathbb{L}^{-1}, r s \mathbb{L}^{-2}\right) \\
& +(\mathbb{L}-1)^{4} \Psi\left(t b d, a c, s \mathbb{L}^{-1}, r \mathbb{L}^{-1}, p q \mathbb{L}^{-2}\right) \\
& +(\mathbb{L}-1)^{4} \Psi\left(t a c, b d, r \mathbb{L}^{-1}, s \mathbb{L}^{-1}, p q \mathbb{L}^{-2}\right) \\
& +(\mathbb{L}-1)^{5}(\mathbb{L}-2) \Phi\left(t a b c d, 1,1,1 ; \mathbb{L}^{-1} p q, \mathbb{L}^{-1}, \mathbb{L}^{-1} r s, \mathbb{L}^{-1}\right) \\
& +(\mathbb{L}-1) B(t, a b c d, 1,1,1, p q, 1, r s, 1) .
\end{aligned}
$$

Consider a blow up. If $v_{y}>v_{x}$, then $y(t)=x(t) \theta(t)$. Therefore,

$$
\begin{gathered}
\sigma^{*}\left(\gamma_{1} \circ \gamma_{2}\right)=\gamma_{1} \circ \gamma_{2}+v_{x}^{(1)} v_{x}^{(2)}, \quad \sigma^{*} v_{x}^{(1)}=v_{x}^{(1)} \\
\sigma^{*} v_{x}^{(2)}=v_{x}^{(2)}, \quad \sigma^{*} v_{y}^{(1)}=v_{x}^{(1)}+v_{\theta}^{(1)}, \quad \sigma^{*} v_{y}^{(2)}=v_{x}^{(2)}+v_{\theta}^{(2)} .
\end{gathered}
$$


Using the Denef-Loeser change of variables formula, we obtain

$$
\begin{aligned}
& B(t, a, b, c, d, p, q, r, s)=\int_{\mathcal{L}^{(1)} \times \mathcal{L}^{(2)}} t^{\gamma_{1} \circ \gamma_{2}+v_{x}^{(1)} v_{x}^{(2)}} a^{v_{x}^{(1)}} v_{x}^{(2)} b^{v_{x}^{(1)} v_{x}^{(2)}+v_{x}^{(1)} v_{\theta}^{(2)}} c^{v_{x}^{(1)} v_{x}^{(2)}+v_{\theta}^{(1)} v_{x}^{(2)}} \\
& \times d^{v_{x}^{(1)}} v_{x}^{(2)}+v_{\theta}^{(1)} v_{x}^{(2)}+v_{x}^{(1)} v_{\theta}^{(2)}+v_{\theta}^{(1)} v_{\theta}^{(2)} p^{v_{x}^{(1)}} q^{v_{x}^{(1)}+v_{\theta}^{(1)}} r_{x}^{v_{x}^{(2)}} s^{v_{x}^{(2)}+v_{\theta}^{(2)}} \mathbb{L}^{-v_{x}^{(1)}-v_{x}^{(2)}} d \chi_{g} \\
& =J\left(t, t a b c d, b d, c d, d, p q \mathbb{L}^{-1}, q, r s \mathbb{L}, s\right) .
\end{aligned}
$$

Substituting this expression for $B(\cdot)$ in (8), we arrive at the following statement.

Lemma 4. The function $J$ satisfies the functional equation

$$
\begin{aligned}
J(t, a, b, c, d, p, q, r, s)= & J\left(t, t a b c d, b d, c d, d, p q \mathbb{L}^{-1}, q, r s \mathbb{L}^{-1}, s\right) \\
& +(\mathbb{L}-1)^{4} \Phi\left(b t, a, d, c, \mathbb{L}^{-1} p, \mathbb{L}^{-1} q, \mathbb{L}^{-1} s, \mathbb{L}^{-1} r\right) \\
& +(\mathbb{L}-1)^{4} \Psi\left(t a b, c d, p \mathbb{L}^{-1}, q \mathbb{L}^{-1}, r s \mathbb{L}^{-2}\right) \\
& +(\mathbb{L}-1)^{4} \Phi\left(c t, d, a, b, q \mathbb{L}^{-1}, p \mathbb{L}^{-1}, r \mathbb{L}^{-1}, s \mathbb{L}^{-1}\right) \\
& +J\left(t, t a b c d, a c, a b, a, p q \mathbb{L}^{-1}, p, r s \mathbb{L}^{-1}, r\right) \\
& +(\mathbb{L}-1)^{4} \Psi\left(t c d, a b, q \mathbb{L}^{-1}, p \mathbb{L}^{-1}, r s \mathbb{L}^{-2}\right) \\
& +(\mathbb{L}-1)^{4} \Psi\left(t b d, a c, s \mathbb{L}^{-1}, r \mathbb{L}^{-1}, p q \mathbb{L}^{-2}\right) \\
& +(\mathbb{L}-1)^{4} \Psi\left(t a c, b d, r \mathbb{L}^{-1}, s \mathbb{L}^{-1}, p q \mathbb{L}^{-2}\right) \\
& +(\mathbb{L}-1)^{5}(\mathbb{L}-2) \Phi\left(t a b c d, 1,1,1 ; \mathbb{L}^{-1} p q, \mathbb{L}^{-1}, \mathbb{L}^{-1} r s, \mathbb{L}^{-1}\right) \\
& +(\mathbb{L}-1) J\left(t, t a b c d, 1,1,1, p q \mathbb{L}^{-1}, 1, r s \mathbb{L}^{-1}, 1\right) .
\end{aligned}
$$

\section{§6. Power structures}

The notion of a power structure over a (semi)ring was introduced by Gusein-Zade, Luengo, and Melle-Hernández in [2].

Definition. A power structure on the ring $R$ is a map

$$
(1+t R[[t]]) \times R \rightarrow 1+t R[[t]]:(A(t), m) \mapsto(A(t))^{m}
$$

satisfying the following properties:

1. $(A(t))^{0}=1$,

2. $(A(t))^{1}=A(t)$,

3. $(A(t) \cdot B(t))^{m}=(A(t))^{m} \cdot(B(t))^{m}$,

4. $(A(t))^{m+n}=(A(t))^{m} \cdot(A(t))^{n}$,

5. $(A(t))^{m n}=\left((A(t))^{n}\right)^{m}$,

6. $(1+t)^{m}=1+m t+$ terms of higher degree,

7. $\left(A\left(t^{k}\right)\right)^{m}=\left.\left((A(t))^{m}\right)\right|_{t \rightarrow t^{k}}$.

A power structure is said to be finitely determined if for every $N>0$ there exists $M>0$ such that the $N$-jet of the series $(A(t))^{m}$ is uniquely determined by the $M$-jet of the series $A(t)$.

To fix a finitely determined power structure, it suffices to define the series $(1-t)^{-m}$ for every $m \in R$ so that $(1-t)^{-m-n}=(1-t)^{-m} \cdot(1-t)^{-n}$. The Grothendieck ring of varieties admits a power structure such that

$$
(1-t)^{-[X]}=1+\left[S^{1} X\right] t+\left[S^{2} X\right] t^{2}+\cdots,
$$

where $S^{k} X=X^{k} / S_{k}$ denotes the $k$ th symmetric power of $X$. For example, for $j \geq 0$ we have

$$
(1-t)^{-\mathbb{L}^{j}}=\sum_{k=0}^{\infty} t^{k} \mathbb{L}^{k j}=\left(1-t \mathbb{L}^{j}\right)^{-1} .
$$


For $X \in K_{0}\left(\operatorname{Var}_{\mathbb{C}}\right), k>0$, put

$$
(1-t)^{-\mathbb{L}^{-k} X}=\left.(1-u)^{-X}\right|_{u=\mathbb{L}^{-k} t} .
$$

The following statement determines the corresponding power structure over the ring $\mathcal{M}$.

Lemma 5. The map $K_{0}\left(\operatorname{Var}_{\mathbb{C}}\right)\left[\mathbb{L}^{-1}\right] \rightarrow 1+t K_{0}\left(\operatorname{Var}_{\mathbb{C}}\right)\left[\mathbb{L}^{-1}\right][[t]]$,

$$
Z \mapsto(1-t)^{-Z}
$$

is well defined. It transforms the sum to the product and is continuous with respect to the filtration $F_{k}$.

We are going to construct a measure on the symmetric power $S^{k} \mathcal{L}$. For a cylindric set $A=\pi_{n}^{-1}\left(A_{n}\right)$, we put $\mu\left(S^{k} A\right)=\mathbb{L}^{-2 n k}\left[S^{k} A_{n}\right]$. This construction agrees with the power structure over the $\operatorname{ring} \mathcal{M}$, so that

$$
\sum_{k} \mu\left(S^{k} A\right) t^{k}=(1-t)^{-\mu(A)} .
$$

Let $B_{i}$ be a collection of nonintersecting cylindric subsets of $\mathcal{L}$, and let $k_{i}$ be nonnegative integers with $\sum k_{i}=n$. Then for the natural embedding

$$
S^{k_{1}} B_{1} \times S^{k_{2}} B_{2} \times \cdots \rightarrow S^{n} \mathcal{L}
$$

we put $\mu\left(\prod_{i} S^{k_{i}} B_{i}\right)=\prod_{i} \mu\left(S_{k_{i}} B_{i}\right)$. Consider the algebra of sets generated by such products of symmetric powers of cylindric sets. The extension of $\mu$ is a well-defined additive measure on this algebra.

Lemma 6. Let $f$ be a simple function on $\mathcal{L}$. Let $F$ be the function on $\bigsqcup_{k} S^{k} \mathcal{L}$ defined by the formula $F\left(\gamma_{1}, \ldots, \gamma_{k}\right)=\prod_{i} f\left(\gamma_{i}\right)$. Then

$$
\int_{\bigsqcup_{k} S^{k} \mathcal{L}} F d \chi_{g}=\int_{\mathcal{L}}(1-f)^{-d \chi_{g}} .
$$

Here $d \chi_{g}$ is in the exponent to emphasize that $1-f$ is viewed as an element of an Abelian group written multiplicatively.

Proof. Consider $\mathcal{L}=\bigsqcup_{j} B_{j},\left.f\right|_{B_{j}}=f_{j}$. Then

$$
S^{k} \mathcal{L}=\bigsqcup_{k_{1}+k_{2}+\cdots=k} S^{k_{1}} B_{1} \times S^{k_{2}} B_{2} \times \cdots
$$

and

$$
\left.F\right|_{S^{k_{1}} B_{1} \times S^{k_{2}} B_{2} \times \cdots}=f_{1}^{k_{1}} \cdot f_{2}^{k_{2}} \cdots
$$

Therefore,

$$
\begin{aligned}
\int_{\bigsqcup_{k} S^{k} \mathcal{L}} & =\sum_{k} \sum_{\sum k_{i}=k} \mu\left(S^{k_{1}} B_{1} \times S^{k_{2}} B_{2} \times \cdots\right) f_{1}^{k_{1}} f_{2}^{k_{2}} \cdots \\
& =\prod_{j}\left(1+\mu\left(S^{1} B_{j}\right) f_{j}+\mu\left(S^{2} B_{j}\right) f_{j}^{2}+\cdots\right) \\
& =\prod_{j}\left(1-f_{j}\right)^{-\mu\left(B_{j}\right)}=\int_{\mathcal{L}}(1-f)^{-d \chi_{g}}
\end{aligned}
$$


§7. An equation for the intersection numbers on $\mathcal{L} \times \bigsqcup_{k} S^{k} \mathcal{L}$

Consider the generating function

$I(t, a, b, c, d, p, q, r, s, u)$

$$
=\int_{\mathcal{L} \times \bigsqcup_{k} S^{k} \mathcal{L}} t^{\gamma_{1} \circ \gamma_{2}} a^{v_{x}^{(1)}} v_{x}^{(2)} b_{x}^{v_{x}^{(1)}} v_{y}^{(2)} c^{v_{y}^{(1)}} v_{x}^{(2)} d^{v_{y}^{(1)}} v_{y}^{(2)} p^{v_{x}^{(1)}} q_{y}^{v_{y}^{(1)}} r_{x}^{v_{x}^{(2)}} s^{v_{y}^{(2)}} u^{k} d \chi_{g} .
$$

In this section we obtain a functional equation for the function $I$.

Let

$$
\prod_{k \geq l}\left(1-x^{k} y^{l} u\right)^{-(\mathbb{L}-1)^{2}}=\sum_{k_{1}, k_{2}} \varepsilon_{k_{1}, k_{2}}(u) x^{k_{1}} y^{k_{2}},
$$

and let

$$
\begin{aligned}
\prod_{k<l} & \left(1-(x y)^{k} z^{l} u\right)^{-(\mathbb{L}-1)^{2}} \prod_{k>l}\left(1-x^{k}(y z)^{l} u\right)^{-(\mathbb{L}-1)^{2}} \prod_{k<l}\left(1-(x y z)^{k} \mathbb{L}^{-l} u\right)^{-(\mathbb{L}-2)(\mathbb{L}-1)^{2}} \\
& =\sum_{k_{1}, k_{2}, k_{3}} \alpha_{k_{1}, k_{2}, k_{3}}(u) x^{k_{1}} y^{k_{2}} z^{k_{3}} .
\end{aligned}
$$

We consider

$$
\begin{aligned}
J_{\gamma_{1}}(t, a, b, c, d, r, s, u) & =\int_{\bigsqcup_{k} S^{k} \mathcal{L}} t^{\gamma_{1} \circ \gamma_{2}} a^{v_{x}^{(1)} v_{x}^{(2)}} b^{v_{x}^{(1)}} v_{y}^{(2)} c^{v_{y}^{(1)}} v_{x}^{(2)} d^{v_{y}^{(1)}} v_{y}^{(2)} r^{v_{x}^{(2)}} s^{v_{y}^{(2)}} u^{k} d \chi_{g} \\
& =\int_{\mathcal{L}^{(2)}}\left(1-t^{\gamma_{1} \circ \gamma_{2}} a^{v_{x}^{(1)}} v_{x}^{(2)} b^{v_{x}^{(1)}} v_{y}^{(2)} c^{v_{y}^{(1)}} v_{x}^{(2)} d^{v_{y}^{(1)}} v_{y}^{(2)} r^{v_{x}^{(2)}} s^{v_{y}^{(2)}} u\right)^{-d \chi_{g}^{(2)}} .
\end{aligned}
$$

Then

$$
I(t, a, b, c, d, p, q, r, s, u)=\int_{\mathcal{L}^{(1)}} p^{v_{x}^{(1)}} q^{v_{y}^{(1)}} J_{\gamma_{1}}(t, a, b, c, d, r, s, u) d \chi_{g}^{(1)} .
$$

If $v_{y}^{(1)}>v_{x}^{(1)}$, then

$$
\begin{aligned}
& J_{\gamma_{1}}(t, a, b, c, d, r, s, u) \\
& =\int_{\left\{v_{y}^{(2)}>v_{x}^{(2)}\right\}}\left(1-t^{\gamma_{1} \circ \gamma_{2}} a^{v_{x}^{(1)}} v_{x}^{(2)} b_{x}^{v_{x}^{(1)} v_{y}^{(2)}} c^{v_{y}^{(1)}} v_{x}^{(2)} d^{v_{y}^{(1)}} v_{y}^{(2)} r_{x}^{v_{x}^{(2)}} s_{y}^{v_{y}^{(2)}} u\right)^{-d \chi_{g}^{(2)}} \\
& \quad \times \int_{\left\{v_{y}^{(2)} \leq v_{x}^{(2)}\right\}}\left(1-t^{v_{x}^{(1)}} v_{y}^{(2)} a^{v_{x}^{(1)}} v_{x}^{(2)} b^{v_{x}^{(1)}} v_{y}^{(2)} c^{v_{y}^{(1)}} v_{x}^{(2)} d^{v_{y}^{(1)}} v_{y}^{(2)} r_{x}^{v_{x}^{(2)}} s_{y}^{v^{(2)}} u\right)^{-d \chi_{g}^{(2)}} .
\end{aligned}
$$

We blow up the origin. The Denef-Loeser formula shows that $d \chi_{g}^{(2)} \mapsto \mathbb{L}^{-v_{x}^{(2)}} d \chi_{g}^{(2)}$. Therefore, the first integral is equal to

$$
\begin{array}{r}
\int_{\mathcal{L}^{(2)}}\left(1-t^{\sigma^{-1}\left(\gamma_{1}\right) \circ \gamma_{2}}(t a b c d)^{\widetilde{v_{x}}} v_{x}^{(2)}(b d)^{\widetilde{v_{x}}(1)} v_{y}^{(2)}(c d)^{\widetilde{v_{y}}}\left(v_{x}^{(2)} d^{\widetilde{v_{y}}} v_{y}^{(1)}(r s)^{v_{x}^{(2)}} s_{y}^{v_{y}^{(2)}} u\right)^{-\mathbb{L}^{-v_{x}^{(2)}} d \chi_{g}^{(2)}}\right. \\
=J_{\sigma^{-1}\left(\gamma_{1}\right)}\left(t, t a b c d, b d, c d, d, r s \mathbb{L}^{-1}, s, u\right) .
\end{array}
$$

The second integral is equal to

$$
\begin{aligned}
\prod_{k \leq l}\left(1-a^{l v_{x}^{(1)}}(b t)^{k v_{x}^{(1)}} c^{l v_{y}^{(1)}} d^{k v_{y}^{(1)}} r^{l} s^{k} u\right)^{-\mathbb{L}^{-k-l}(\mathbb{L}-1)^{2}} & \\
= & \prod_{k \leq l}\left(1-a^{l v_{x}^{(1)}}(b t)^{k v_{x}^{(1)}} c^{l v_{y}^{(1)}} d^{k v_{y}^{(1)}}\left(\mathbb{L}^{-1} r\right)^{l}\left(\mathbb{L}^{-1} s\right)^{k} u\right)^{-(\mathbb{L}-1)^{2}} \\
& =\sum_{k_{1}, k_{2}} \varepsilon_{k_{1}, k_{2}}(u)\left(\mathbb{L}^{-1} r\right)^{k_{1}}\left(\mathbb{L}^{-1} s\right)^{k_{2}} a^{k_{1} v_{x}^{(1)}}(b t)^{k_{2} v_{x}^{(1)}} c^{k_{1} v_{y}^{(1)}} d^{k_{2} v_{y}^{(1)}} .
\end{aligned}
$$


Hence,

$$
\begin{aligned}
J_{\gamma_{1}}(t, a, b, c, d, r, s, u) & =J_{\sigma^{-1}\left(\gamma_{1}\right)}\left(t, t a b c d, b d, c d, d, r s \mathbb{L}^{-1}, s, u\right) \\
\times & \left(\sum_{k_{1}, k_{2}} \varepsilon_{k_{1}, k_{2}}(u)\left(\mathbb{L}^{-1} r\right)^{k_{1}}\left(\mathbb{L}^{-1} s\right)^{k_{2}} a^{k_{1} v_{x}^{(1)}}(b t)^{k_{2} v_{x}^{(1)}} c^{k_{1} v_{y}^{(1)}} d^{k_{2} v_{y}^{(1)}}\right) .
\end{aligned}
$$

Thus, we see that

$$
\begin{aligned}
& E(t, a, b, c, d, p, q, r, s, u) \\
& =\int_{\left\{v_{y}^{(1)}>v_{x}^{(1)}\right\}} t^{\gamma_{1} \circ \gamma_{2}} a^{v_{x}^{(1)} v_{x}^{(2)}} b_{x}^{v_{x}^{(1)} v_{y}^{(2)}} c^{v_{y}^{(1)} v_{x}^{(2)}} d^{v_{y}^{(1)}} v_{y}^{(2)} p^{v_{x}^{(1)}} q^{v_{y}^{(1)}} r_{x}^{v_{x}^{(2)}} s_{y}^{v_{y}^{(2)}} u^{k} d \chi_{g} \\
& =\int_{\left\{v_{y}^{(1)}>v_{x}^{(1)}\right\}} J_{\gamma_{1}}(t, a, b, c, d, r, s, u) p^{v_{x}^{(1)}} q^{v_{y}^{(1)}} d \chi_{g}^{(1)} \\
& =\sum_{k_{1}, k_{2}} \varepsilon_{k_{1}, k_{2}}(u)\left(\mathbb{L}^{-1} r\right)^{k_{1}}\left(\mathbb{L}^{-1} s\right)^{k_{2}} \int_{\left\{v_{y}^{(1)}>v_{x}^{(1)}\right\}} J_{\sigma^{-1}\left(\gamma_{1}\right)}\left(t, t a b c d, b d, c d, d, r s \mathbb{L}^{-1}, s, u\right) \\
& \times a^{k_{1} v_{x}^{(1)}}(b t)^{k_{2} v_{x}^{(1)}} c^{k_{1} v_{y}^{(1)}} d^{k_{2} v_{y}^{(1)}} p^{v_{x}^{(1)}} q^{v_{y}^{(2)}} d \chi_{g}^{(1)} \\
& =\sum_{k_{1}, k_{2}} \varepsilon_{k_{1}, k_{2}}(u)\left(\mathbb{L}^{-1} r\right)^{k_{1}}\left(\mathbb{L}^{-1} s\right)^{k_{2}} \int_{\left\{v_{y}^{(1)}>v_{x}^{(1)}\right\}} J_{\sigma^{-1}\left(\gamma_{1}\right)}\left(t, t a b c d, b d, c d, d, r s \mathbb{L}^{-1}, s, u\right) \\
& \times\left(a^{k_{1}}(b t)^{k_{2}} p\right)^{v_{x}^{(1)}}\left(c^{k_{1}} d^{k_{2}} q\right)^{v_{y}^{(1)}} d \chi_{g}^{(1)} \\
& =\sum_{k_{1}, k_{2}} \varepsilon_{k_{1}, k_{2}}(u)\left(\mathbb{L}^{-1} r\right)^{k_{1}}\left(\mathbb{L}^{-1} s\right)^{k_{2}} \\
& \times \int_{\mathcal{L}^{(1)}} J_{\gamma_{1}}\left(t, t a b c d, b d, c d, d, r s \mathbb{L}^{-1}, s, u\right)\left((a c)^{k_{1}}(b d t)^{k_{2}} p q \mathbb{L}^{-1}\right)^{v_{x}^{(1)}}\left(c^{k_{1}} d^{k_{2}} q\right)^{v_{y}^{(1)}} d \chi_{g}^{(1)} \\
& =\sum_{k_{1}, k_{2}} \varepsilon_{k_{1}, k_{2}}(u)\left(\mathbb{L}^{-1} r\right)^{k_{1}}\left(\mathbb{L}^{-1} s\right)^{k_{2}} \\
& \times I\left(t, t a b c d, b d, c d, d,(a c)^{k_{1}}(b d t)^{k_{2}} p q \mathbb{L}^{-1}, c^{k_{1}} d^{k_{2}} q, r s \mathbb{L}^{-1}, s, u\right) .
\end{aligned}
$$

Clearly, the same integral over $\left\{v_{x}^{(1)}<v_{y}^{(1)}\right\}$ is equal to $E(t, d, c, b, a, q, p, s, r, u)$.

We compute the integral over $\left\{v_{x}^{(1)}=v_{y}^{(1)}\right\}$. Let $y^{(1)}=\lambda_{1} x^{(1)}+\widetilde{y}^{(1)}$. Then

$$
\begin{aligned}
& J_{\gamma_{1}}(t, a, b, c, d, r, s, u) \\
& =\int_{\left\{v_{x}^{(2)}<v_{y}^{(2)}\right\}}\left(1-t^{\gamma_{1} \circ \gamma_{2}} a^{v_{x}^{(1)} v_{x}^{(2)}} b^{v_{x}^{(1)} v_{y}^{(2)}} c^{v_{y}^{(1)} v_{x}^{(2)}} d^{v_{y}^{(1)}} v_{y}^{(2)} r_{x}^{v_{x}^{(2)}} s_{y}^{v_{y}^{(2)}} u\right)^{-d \chi_{g}^{(2)}} \\
& \times \int_{\left\{v_{x}^{(2)}>v_{y}^{(2)}\right\}}\left(1-t^{\gamma_{1} \circ \gamma_{2}} a^{v_{x}^{(1)}} v_{x}^{(2)} b^{v_{x}^{(1)} v_{y}^{(2)}} c^{v_{y}^{(1)} v_{x}^{(2)}} d_{y}^{v_{y}^{(1)} v_{y}^{(2)}} r^{v_{x}^{(2)}} s^{v_{y}^{(2)}} u\right)^{-d \chi_{g}^{(2)}} \\
& \times \int_{\left\{v_{x}^{(2)}=v_{y}^{(2)}\right\}}\left(1-t^{\gamma_{1} \circ \gamma_{2}} a^{v_{x}^{(1)}} v_{x}^{(2)} b^{v_{x}^{(1)}} v_{y}^{(2)} c^{v_{y}^{(1)} v_{x}^{(2)}} d^{v_{y}^{(1)} v_{y}^{(2)}} r^{v_{x}^{(2)}} s^{v_{y}^{(2)}} u\right)^{-d \chi_{g}^{(2)}} \\
& =\prod_{k<l}\left(1-(t a c)^{k v_{x}^{(1)}}(b d)^{l v_{x}^{(1)}} r^{k} s^{l} u\right)^{-\mathbb{L}^{-k-l}(\mathbb{L}-1)^{2}} \\
& \times \prod_{k>l}\left(1-(a c)^{k v_{x}^{(1)}}(t b d)^{l v_{x}^{(1)}} r^{k} s^{l} u\right)^{-\mathbb{L}^{-k-l}(\mathbb{L}-1)^{2}} \\
& \times \int_{\left\{v_{x}^{(2)}<v_{y}^{(2)}\right\}}\left(1-t^{\gamma_{1} \circ \gamma_{2}} a^{v_{x}^{(1)} v_{x}^{(2)}} b_{x}^{v_{x}^{(1)} v_{y}^{(2)}} c^{v_{y}^{(1)}} v_{x}^{(2)} d^{v_{y}^{(1)} v_{y}^{(2)}} r^{v_{x}^{(2)}} s^{v_{y}^{(2)}} u\right)^{-d \chi_{g}^{(2)}} .
\end{aligned}
$$


The last integral can be decomposed into the product of integrals over $\left\{\lambda_{2} \neq \lambda_{1}\right\}$ and $\left\{\lambda_{2}=\lambda_{1}\right\}$. The integral over $\left\{\lambda_{2} \neq \lambda_{1}\right\}$ is equal to

$$
\left[\prod_{k<l}\left(1-(t a b c d)^{k v_{x}^{(1)}}(r s)^{k} u\right)^{-\mathbb{L}^{-k-l}(\mathbb{L}-1)^{2}}\right]^{(\mathbb{L}-2)} .
$$

If $\lambda_{1}=\lambda_{2}=\lambda$, we can make the affine change of variables $A_{\lambda}:(x, y) \mapsto(x, y-\lambda x)$. Therefore, this integral is equal to

$$
\begin{array}{r}
\int_{\left\{v_{\tilde{y}}^{(2)}>v_{x}^{(2)}\right\}}\left(1-t^{A_{\lambda}\left(\gamma_{1}\right) \circ \gamma_{2}}(a b c d)^{v_{x}^{(1)}}(r s)^{v_{x}^{(2)}} u\right)^{-d \chi_{g}^{(2)}} \\
=J_{\sigma^{-1}\left(A_{\lambda}\left(\gamma_{1}\right)\right)}\left(t, t a b c d, 1,1,1, r s \mathbb{L}^{-1}, 1, u\right) .
\end{array}
$$

The product of the remaining factors is equal to

$$
\begin{aligned}
\prod_{k<l}(1- & \left.\left(t^{v_{x}^{(1)}} \cdot(a c)^{v_{x}^{(1)}} r \mathbb{L}^{-1}\right)^{k}\left((b d)^{v_{x}^{(1)}} s \mathbb{L}^{-1}\right)^{l} u\right)^{-1} \\
& \times \prod_{k>l}\left(1-\left((a c)^{v_{x}^{(1)}} r \mathbb{L}^{-1}\right)^{k}\left(t^{v_{x}^{(1)}} \cdot(b d)^{v_{x}^{(1)}} s \mathbb{L}^{-1} u\right)^{l}\right)^{-1} \\
& \times \prod_{k<l}\left(1-\left(t^{v_{x}^{(1)}} \cdot(a c)^{v_{x}^{(1)}} r \mathbb{L}^{-1} \cdot(b d)^{v_{x}^{(1)}} s \mathbb{L}^{-1}\right)^{k} \mathbb{L}^{-l} u\right)^{-(\mathbb{L}-2)} \\
= & \sum_{k_{1}, k_{2}, k_{3}} \alpha_{k_{1}, k_{2}, k_{3}}(u) t^{k_{1} v_{x}^{(1)}}(a c)^{k_{2} v_{x}^{(1)}}\left(r \mathbb{L}^{-1}\right)^{k_{2}}(b d)^{k_{3} v_{x}^{(1)}}\left(s \mathbb{L}^{-1}\right)^{k_{3}} .
\end{aligned}
$$

Consequently,

$$
\begin{aligned}
\int_{\left\{v_{x}^{(1)}=v_{y}^{(1)}\right\}} & J_{\gamma_{1}}(t, a, b, c, d, r, s, u) p^{v_{x}^{(1)}} q_{x}^{v_{x}^{(1)}} d \chi_{g}^{(1)} \\
= & \int_{\mathbb{C}^{*}} d \chi_{g}(\lambda) \int_{\left\{y^{(1)}=\lambda x^{(1)}+\widetilde{y}^{(1)}\right\}} \sum_{k_{1}, k_{2}, k_{3}} \alpha_{k_{1}, k_{2}, k_{3}}(u)\left(r \mathbb{L}^{-1}\right)^{k_{2}}\left(s \mathbb{L}^{-1}\right)^{k_{3}} \\
& \times J_{\sigma^{-1}\left(A_{\lambda}\left(\gamma_{1}\right)\right)}\left(t, t a b c d, 1,1,1, r s \mathbb{L}^{-1}, 1, u\right)\left(t^{k_{1}}(a c)^{k_{2}}(b d)^{k_{3}} p q\right)^{v_{x}^{(1)}} d \chi_{g}^{(1)} \\
= & (\mathbb{L}-1) \int_{\left\{v_{\tilde{y}}^{(1)}>v_{x}^{(1)}\right\}} \sum_{k_{1}, k_{2}, k_{3}} \alpha_{k_{1}, k_{2}, k_{3}}(u)\left(r \mathbb{L}^{-1}\right)^{k_{2}}\left(s \mathbb{L}^{-1}\right)^{k_{3}} \\
\quad & \times J_{\sigma^{-1}\left(\gamma_{1}\right)}\left(t, t a b c d, 1,1,1, r s \mathbb{L}^{-1}, 1, u\right)\left(t^{k_{1}}(a c)^{k_{2}}(b d)^{k_{3}} p q\right)^{v_{x}^{(1)}} d \chi_{g}^{(1)} \\
= & \mathbb{L}-1) \int_{\mathcal{L}^{(1)}} \sum \alpha_{k_{1}, k_{2}, k_{3}}(u)\left(r \mathbb{L}^{-1}\right)^{k_{2}}\left(s \mathbb{L}^{-1}\right)^{k_{3}} \\
& \times J_{\gamma_{1}}\left(t, t a b c d, 1,1,1, r s \mathbb{L}^{-1}, 1, u\right)\left(t^{k_{1}}(a c)^{k_{2}}(b d)^{k_{3}} p q \mathbb{L}^{-1}\right)^{v_{x}^{(1)}} d \chi_{g}^{(1)} \\
= & (\mathbb{L}-1) \sum \alpha_{k_{1}, k_{2}, k_{3}}(u)\left(r \mathbb{L}^{-1}\right)^{k_{2}}\left(s \mathbb{L}^{-1}\right)^{k_{3}} \\
& \times I\left(t, t a b c d, 1,1,1, t^{k_{1}}(a c)^{k_{2}}(b d)^{k_{3}} p q, 1, r s \mathbb{L}^{-1}, 1, u\right) .
\end{aligned}
$$

Combining these integrals, we arrive at the following statement. 


\section{Theorem 4.}

$$
\begin{aligned}
& I(t, a, b, c, d, p, q, r, s, u) \\
& =\sum_{k_{1}, k_{2}} \varepsilon_{k_{1}, k_{2}}(u)\left(\mathbb{L}^{-1} r\right)^{k_{1}}\left(\mathbb{L}^{-1} s\right)^{k_{2}} \\
& \quad \times I\left(t, t a b c d, b d, c d, d,(a c)^{k_{1}}(b d t)^{k_{2}} p q \mathbb{L}^{-1}, c^{k_{1}} d^{k_{2}} q, r s \mathbb{L}^{-1}, s, u\right) \\
& +\sum_{k_{1}, k_{2}} \varepsilon_{k_{1}, k_{2}}(u)\left(\mathbb{L}^{-1} s\right)^{k_{1}}\left(\mathbb{L}^{-1} r\right)^{k_{2}} \\
& \quad \times I\left(t, t a b c d, a c, a b, a,(b d)^{k_{1}}(a c t)^{k_{2}} p q \mathbb{L}^{-1}, b^{k_{1}} a^{k_{2}} p, r s \mathbb{L}^{-1}, r, u\right) \\
& +(\mathbb{L}-1) \sum \alpha_{k_{1}, k_{2}, k_{3}}(u)\left(\mathbb{L}^{-1} r\right)^{k_{2}}\left(\mathbb{L}^{-1} s\right)^{k_{3}} \\
& \quad \times I\left(t, t a b c d, 1,1,1, t^{k_{1}}(a c)^{k_{2}}(b d)^{k_{3}} p q, 1, r s \mathbb{L}^{-1}, 1, u\right) .
\end{aligned}
$$

\section{ACKNOWLEDGEMENTS}

I would like to thank S. Gusein-Zade for permanent attention, encouragement, and useful discussions.

\section{REFERENCES}

[1] J. Denef and F. Loeser, Germs of arcs on singular algebraic varieties and motivic integration, Invent. Math. 135 (1999), no. 1, 201-232. MR1664700(99k:14002)

[2] S. M. Gusein-Zade, I. Luengo, and A. Melle-Hernández, A power structure over the Grothendieck ring of varieties, Math. Res. Lett. 11 (2004), no. 1, 49-57. MR2046199 (2004m:14038)

[3] M. Kapranov, The elliptic curve in the S-duality theory and Eisenstein series for Kac-Moody groups, arXiv: math.AG/0001005.

[4] F. Heinloth, A note on functional equations for zeta functions with values in Chow motives, Ann. Inst. Fourier (Grenoble) 57 (2007), 1927-1945. arXiv: math.AG/0512237. MR2377891

[5] V. I. Arnol'd, A. N. Varchenko, and S. M. Guseı̆n-Zade, Singularities of differentiable maps. Vol. II. Monodromy and asymptotics of integrals, "Nauka", Moscow, 1984; English transl., Monogr. Math., vol. 83, Birkhäuser Boston, Inc., Boston, MA, 1988. MR0755329 (86m:58026) MR0966191 (89g:58024)

Moscow State University and Independent University of Moscow, Russia

E-mail address: gorsky@mccme.ru

Received 3/OCT/2006

Translated by THE AUTHOR 